\title{
1 Estimating airborne heavy metal concentrations in Dunkerque (Northern France)
}

2

3 Germán Santos $^{1 *}$, Ignacio Fernández-Olmo ${ }^{1}$, Ángel Irabien ${ }^{1}$, Frédéric Ledoux ${ }^{2}$, Dominique Courcot $^{2}$

4

5 'Departamento de Ingenierías Química y Biomolecular, Universidad de Cantabria, Santander, 39005, Spain

6

$7 \quad{ }^{2}$ Unité de Chimie Environnementale et Interactions sur le Vivant (UCEIV) EA 4492, Université du Littoral

8 Côte d'Opale, Dunkerque, 59140, France

9

10 *Corresponding author. Tel.: +34 942201579; fax: +34 942201591

11 E-mail address: santosg@unican.es

\section{Abstract}

14 This work aims to estimate the levels of lead $(\mathrm{Pb})$, nickel $(\mathrm{Ni})$, manganese $(\mathrm{Mn})$, vanadium $(\mathrm{V})$ and 15 chromium $(\mathrm{Cr})$ corresponding to a three-month $\mathrm{PM}_{10}$ sampling campaign conducted in 2008 in the city of 16 Dunkerque (Northern France) by means of statistical models based on Partial Least Squares Regression 17 (PLSR), Artificial Neural Networks (ANN) and Principal Component Analysis (PCA) coupled with ANN. 18 According to the European Air Quality Directives, because the levels of these pollutants are sufficiently below the European Union (EU) limit/target values and other air quality guidelines, they may be used for air quality assessment purposes as an alternative to experimental measurements. An external validation of the models has been conducted, and the results indicate that PLSR and ANNs, with comparable performance, provide adequate mean concentration estimations for $\mathrm{Pb}, \mathrm{Ni}, \mathrm{Mn}$ and $\mathrm{V}$, fulfilling the $\mathrm{EU}$ uncertainty requirements for objective estimation techniques, although ANNs seem to present better generalization ability. However, in accordance with the European regulation, both techniques can be considered acceptable air quality assessment tools for heavy metals in the studied area. Furthermore, the application of factor analysis prior to ANNs did not yield any improvements in the performance of the ANNs. 


\section{Introduction}

31 Recent studies have shown a positive correlation between high concentrations of particles and public health

32 deterioration. Particulate Matter (PM) remains a concerning environmental problem in urban areas due to

33 its physical properties, such as mass distribution, particle size and shape, and chemical composition, which

34 may include various acidic and toxic species such as metals, metalloids and aromatic compounds (Karar and Gupta 2006). In addition to industrial emissions, non-exhaust PM emissions from road traffic have

36 been identified as an important source of metals in urban environments (Thorpe and Harrison 2008).

37 Furthermore, long-term exposure to metals could cause severe toxic effects on human health (Chen and 38 Lippmann 2009).

39 In this context, the European Union, through the Air Quality Framework Directive (EC 2008) and the $4^{\text {th }}$ 40 Daughter Directive (EC 2004), has established a set of air quality objectives for certain pollutants in $\mathrm{PM}_{10}$ : 41 a limit value of $500 \mathrm{ng} \mathrm{m}^{-3}$ for $\mathrm{Pb}$ (Directive 2008/50/EC) and target values of $6 \mathrm{ng} \mathrm{m}^{-3}$ for $\mathrm{As}, 20 \mathrm{ng} \mathrm{m}^{-3}$ 42 for $\mathrm{Ni}$ and $5 \mathrm{ng} \mathrm{m}^{-3}$ for Cd (Directive 2004/107/EC) for the total content in the $\mathrm{PM}_{10}$ fraction averaged over 43 a calendar year. Along with these limit/target values, an upper and lower assessment threshold (hereafter 44 known as UAT and LAT) are also specified, expressed as a percentage of the corresponding limit/target 45 value as follows: 70 and $50 \%$ ( $\mathrm{Pb}$ and $\mathrm{Ni}$ ) and 60 and $40 \%$ (As and $\mathrm{Cd}$ ). Depending on the level of 46 pollutants with respect to these thresholds, different air quality assessment methods with respect to the 47 pollutants are permitted. Thus, in accordance with Directive 2008/50/EC, when the pollutant levels are 48 below the lower assessment threshold (LAT), the air quality may be assessed using solely modelling or 49 objective estimation techniques without the need for experimental measurements. Taking into account the 50 high cost and time consumption associated with analytical determination of the levels of these pollutants, 51 it may be interesting to try to find new alternatives for air quality assessment so that fewer experimental 52 measurements may be required.

53 According to the Guidance on Assessment under the EU Air Quality Directives, “objective estimation technique" is a fairly broad term that includes mathematical methods to calculate concentrations from values measured at other locations and/or times based on scientific knowledge of the concentration distribution. Empirical data-based modelling or statistical modelling falls within this definition and represents an attractive alternative to deterministic modelling (air dispersion modelling), given that it requires less specific knowledge of the system under consideration as it attempts to find the existing relationship between the immission concentrations of pollutants and other variables that may influence the 
60 processes that control the formation, transportation and removal of aerosols in the atmosphere, disregarding

61 the physical principles in which the equations that describe these processes are based on, as well as other

62 decisive information such as emission inventories.

63 Partial Least Squares Regression and Artificial Neural Networks have been proposed in this study to 64 estimate $\mathrm{PM}_{10}$-bound heavy metals because both have been used in the literature as mathematical 65 techniques to forecast the air concentration of a number of pollutants. Pires et al. (2008), Polat and Durduran 66 (2012) and Singh et al. (2012) applied PLSR to predict PM concentrations, and numerous authors over the 67 years have investigated the development of ANN models to predict PM concentrations and gaseous 68 pollutants (Gardner and Dorling 1999; Kukkonen et al. 2003; Perez and Reyes 2002), to cite but a few. 69 Furthermore, Chelani et al. (2002) performed not only a prediction of $\mathrm{PM}_{10}$ concentration but also of 70 ambient air metal levels, namely $\mathrm{Cd}, \mathrm{Cr}, \mathrm{Fe}, \mathrm{Ni}, \mathrm{Pb}$ and $\mathrm{Zn}$, with a low prediction error. Moreover, because 71 the number of independent input variables is relatively high with respect to the number of samples, an alternative approach based on applying Principal Component Analysis (PCA) prior to ANN development was considered due to this technique being reported in the literature as an effective strategy to improve model performance (Lu et al. 2003, Sousa et al. 2007, Ul-Saufie et al. 2013).

Despite having a relatively small contribution to the total content of PM in terms of mass, metals in Dunkerque have been reported as clear tracers of the local industrial activities in the city (Kfoury 2013). In this respect, the main objective of this work is to estimate the levels of some EU-regulated and nonregulated metals in airborne $\mathrm{PM}_{10}$ in the urban area of Dunkerque. For this purpose, statistical models based on PLSR and ANNs have been developed as objective estimation techniques.

It is worth mentioning that because this work is devised as an air quality assessment tool at a later stage, it

81 is about estimation instead of forecasting. Thus, it is intended to provide an estimation of the pollutant 82 concentrations of the recent past as an alternative to experimental measurements instead of predicting future 83 pollutant concentrations.

\section{2. Description of the methodology and area of investigation}

\subsection{Partial least squares regression fundamentals}

87 Partial least squares regression is a statistical method that, as with other multivariate regression techniques, seeks to find the relationship between two data matrices in order to predict a response or a set of response variables $(Y)$ from a set of predictors $(X)$. However, it differs from other multivariate calibration techniques 
90 in that it aims to reach two goals simultaneously as follows: to capture variance and to achieve correlations,

91 i.e., maximize covariance (Abdi 2010). That is to say, PLSR attempts to find factors that maximize the

92 amount of variation explained in $X$ that is relevant for predicting $Y$ as a generalization of other related techniques, e.g., principal component regression (PCR), which obtains factors based solely on the amount of variance captured in $X$ and disregards entirely the covariance, and multiple linear regression (MLR), which tries to find a single factor that best correlates predictors with responses.

96 By performing a projection of the original predictor variables into a new space, PLSR creates a set of orthogonal factors, referred to as latent variables, to be used to predict the output variable(s). This projection is performed as follows: first, the $X$-matrix is decomposed as a product of a set of $X$-scores $T$ multiplied by a set of $X$-loadings $P$.

$100 X=T P^{\prime}+E$

$101 X$-scores are expressed as a linear combination of the original predictor variables by means of a set of

102 vectors of coefficients known as weights, which ensure the orthogonality of scores.

$103 T=X W^{*}$

104 where

$105 W^{*}=W\left(P^{\prime} W\right)^{-1}$

106 In parallel, a similar decomposition is performed for the $Y$-matrix, which is expressed as a product of the $107 \quad Y$-scores $U$ multiplied by the $Y$-loadings $C$.

$108 Y=U C^{\prime}+G$

109 As mentioned before, $X$-scores not only model $X$ (Eq. (1)) but also predict $Y$. This prediction is achieved 110 using Eq. (5).

$111 Y=T C^{\prime}+F=X W^{*} C^{\prime}+F=X B+F$

112 Therefore

$113 B=W^{*} C^{\prime}$

114 Further details of this technique can be found in Wold (2001). PLS Toolbox (Eigenvector Research, Inc.)

115 for MATLAB was used in the present study to develop the PLSR models.

\section{2.2 Artificial neural network fundamentals}

118 Artificial neural networks are computational systems based on biological nervous systems that attempt to

119 mimic the fault-tolerance and capacity to learn of biological neural systems. They are formed by a number 
120 of highly interconnected simple processing elements, or artificial neurons (also known as nodes or units),

121 receiving a set of inputs, either from original data or from the output of other neurons in the neural network,

122 via weighted connections (or weights) that resemble synaptic connections in a biological neuron. These

123 nodes are arranged into three types of layers, i.e., input, hidden and output layers. Data are fed into the

124 nodes in the input layer and later transferred to the subsequent layers. Every node in the hidden and output

125 layers also has a single bias value known as the activation threshold value. Being the weighted sum of the

126 inputs computed, the corresponding threshold value is subtracted to compose the activation of the neuron.

127 The activation signal is passed through an activation function (also known as a transfer function) to produce

128 the output of the node. The relationship between the output and the inputs finally has the mathematical 129 representation, as presented in Eq. (7):

$130 y_{t}=w_{0}+\sum_{j=1}^{q} w_{j} \cdot g\left(w_{0, j}+\sum_{i=1}^{p} w_{i, j} x_{t, i}\right)+\varepsilon_{t}$

131 where $p$ is the number of input nodes, $q$ is the number of hidden nodes, $w_{i, j}(i=0,1,2, \ldots, p, j=1,2, \ldots, q)$

132 and $w_{j}(j=0,1,2, \ldots, q)$ are connection weights, and $\varepsilon_{t}$ is a bias error.

133 A multitude of neural network architectures are possible. However, in practice, simple network structures

134 with a relatively small number of hidden nodes often work well in out-of-sample forecasting. In this work,

135 a multilayer perceptron (MLP) neural network with a sigmoid hidden transfer function and a linear output

136 transfer function has been selected, applying the Levenberg-Marquardt learning algorithm. A schematic 137 representation of the network structure is shown in Fig. 1.

138 The ANN models in this study were developed using the Neural Network Toolbox for MATLAB 139 (MathWorks, Inc.).

140

\section{$141 \quad 2.3$ Description of the area of study and sampling site}

142 The city of Dunkerque, with a population of approximately 68,000 inhabitants in 2008 , is located on the 143 northern coastline of France, limited by the French-Belgian border. The main urban area is surrounded in 144 its northern part by the harbour of Dunkerque, which is classified as the third most important port in France 145 due to shipping and freight transport (including ore, coal and copper, among other goods) and as the seventh 146 port in order of importance of Northern Europe. The city is also in close proximity to the English Channel, 147 connecting the North Sea with the Atlantic Ocean, which is the world's busiest seaway, with approximately 148500 vessels transiting daily. There is also a highly industrialized area in the city's vicinity for the metallurgic 
149 industry, as it has an integrated steel manufacturing plant (nearly $4 \mathrm{~km} \mathrm{NW})$, an electric steel plant (6 km

$150 \mathrm{NE}$ ) and a ferromanganese alloy production plant (at approximately $6 \mathrm{~km} \mathrm{~W}$ ).

151 A total of 78 samples were measured throughout an intensive $\mathrm{PM}_{10}$ sampling campaign performed from

152 February to May 2008 in Dunkerque by Hleis (2010). Fig. 2 shows the location of the sampling site

$153\left(51^{\circ} 02^{\prime} 07^{\prime \prime} \mathrm{N}, 02^{\circ} 22^{\prime} 05^{\prime}\right.$ 'E and approximately $10 \mathrm{~m}$ above sea level), which was placed on the rooftop of

154 the Les Darses site (to prevent the sampling of punctual events at street / ground level) on the boundary line

155 between the industrial area and the city so that the effects of both urban and industrial emissions were

156 registered during sampling (under WSW and NNW wind sectors) (Kfoury 2013). Further details of the

157 sampling procedure are described in Hleis (2010). The composition of inorganic elements $(\mathrm{Al}, \mathrm{Ca}, \mathrm{Cr}, \mathrm{Cu}$,

$158 \mathrm{Fe}, \mathrm{K}, \mathrm{Mg}, \mathrm{Mn}, \mathrm{Na}, \mathrm{Ni}, \mathrm{Pb}, \mathrm{Sn}, \mathrm{Ti}, \mathrm{V}$ and $\mathrm{Zn})$ and ions $\left(\mathrm{Cl}^{-}, \mathrm{NO}_{3}{ }^{-}, \mathrm{SO}_{4}{ }^{2-}\right.$ and $\left.\mathrm{NH}_{4}{ }^{+}\right)$in the particles was

159 determined. The mean values of these constituents are reported in Hleis (2010).

160

\section{2.4. Modelling database and pre-treatment}

162 As usual for this type of modelling, input variables consist of (i) meteorological data, namely average

163 temperature $\left({ }^{\circ} \mathrm{C}\right)$, average relative humidity $(\%)$, prevailing wind direction $\left({ }^{\circ}\right)$, prevailing wind speed (ms ${ }^{-}$

$164^{1}$ ), average pressure (mbar) and cumulative precipitation $\left(\mathrm{L} \mathrm{m}^{-2}\right)$, which are obtained at the meteorological

165 station in the harbour of Dunkerque, and (ii) major pollutant data, which are composed by average

166 concentrations $\left(\mu \mathrm{g} \mathrm{m} \mathrm{m}^{-3}\right)$ of sulphur dioxide $\left(\mathrm{SO}_{2}\right)$, tropospheric ozone $\left(\mathrm{O}_{3}\right)$ and nitrogen oxides $\left(\mathrm{NO}_{\mathrm{x}}\right)$

167 measured at the St. Pol sur Mer air quality monitoring station (the Atmo-Nord-Pas-de-Calais air quality

168 network) and $\mathrm{PM}_{10}$ concentrations measured at the Les Darses site. Additionally, two nominal variables

169 were considered to account for the seasonal (1: Winter, 2: Spring, 3: Summer, 4: Fall) and weekend effects

170 (0: Working day, 1: Weekend).

171 Output variables in this study consisted of $\mathrm{PM}_{10}$-bound $\mathrm{Pb}, \mathrm{Ni}, \mathrm{Mn}, \mathrm{V}$ and $\mathrm{Cr}$ levels in ambient air (ng $\mathrm{m}^{-3}$ )

172 at the sampling site. Among the EU regulated metals, $\mathrm{Pb}$ and $\mathrm{Ni}$ were determined. Additionally, three non-

173 regulated metals were also considered: $\mathrm{Mn}, \mathrm{V}$ and $\mathrm{Cr}$. These metals were tracers of various industrial

174 activities found in Dunkerque, where previous studies on trace metal levels have been developed: Mn, for

175 ferromanganese alloys manufacturing; $\mathrm{V}$, for marine traffic and liquid fuel combustion; and $\mathrm{Cr}$, for non-

176 integrated steel manufacturing and coal combustion (Kfoury 2013). Because these metals are not regulated

177 by the EU, they do not have a limit/target. Therefore, to normalize the metal concentration and calculate

178 the EU uncertainty indices, the following values were considered as equivalent to the LAT for non- 
179 regulated metals: the annual air quality guideline for $\mathrm{Mn}\left(150 \mathrm{ng} \mathrm{\textrm {m } ^ { - 3 }}\right)$ proposed by the World Health 180 Organization (WHO 2000) and the maximum observed concentration for $\mathrm{V}$ and $\mathrm{Cr}$ in the absence of a 181 standard value for a period of duration comparable with that of the period of study.

182 As shown in Fig. 3, the $\mathrm{Pb}$ and Ni mean values are below their respective LAT. Therefore, according to the

183 EU Air Quality Directives, objective estimation techniques can be applied for the air quality assessment in 184 relation to $\mathrm{Pb}$ and $\mathrm{Ni}$.

185 A pre-treatment procedure for outlier identification and removal based on the statistical parameter of the

186 Mahalanobis distance was conducted. Additionally, as usual for this type of technique, the complete

187 database was divided into three subsets as a result of applying a data-splitting procedure, the Kennard-Stone 188 algorithm, which selects the more representative samples for each subset based on Euclidean distances.

189 Thus, $60 \%$ of the total number of samples was used for model development, $20 \%$ for verification to avoid 190 over-fitting and $20 \%$ for external validation. Furthermore, to avoid scale effects, the dependent variables 191 were normalized by dividing the metal concentrations by their respective LAT.

1932.5 Model performance criteria

194 In this study, the evaluation criteria to determine whether a model is suitable for air quality assessment 195 purposes is principally based on the following: (i) the fulfilment of the European Union uncertainty 196 requirements for objective estimation techniques, and (ii) the accuracy of estimated mean values because 197 the metal limit/target values correspond to annual mean concentrations. Two indices of uncertainty were 198 calculated: the relative maximum error without timing (RME) and the relative directive error (RDE). The

199 former is the largest concentration difference of all percentile ( $p$ ) differences normalized by the respective 200 measured value (Borrego et al. 2008), as calculated by Eq. (8). The latter is the difference between the 201 closest observed concentration to the limit/target value and the correspondingly ranked modelled 202 concentration normalized by the limit/target value (Denby et al. 2010), as given by Eq. (9).

$203 \quad \mathrm{RME}=\max \left(\left|\mathrm{C}_{\mathrm{O}, \mathrm{p}}-\mathrm{C}_{\mathrm{E}, \mathrm{p}}\right|\right) / \mathrm{C}_{\mathrm{O}, \mathrm{p}}$

$204 \quad \mathrm{RDE}=\left|\mathrm{C}_{\mathrm{O}, \mathrm{LV}}-\mathrm{C}_{\mathrm{E}, \mathrm{LV}}\right| / \mathrm{LV}$

205 Additionally, a number of statistical parameters were considered to evaluate the model performance. These 206 performance indicators are the fractional bias (FB), the correlation coefficient $(r)$, the root mean squared 207 error (RMSE) and the fractional variance (FV), as given by Eqs. (10-13):

208 $\mathrm{r}=\left[\frac{\sum_{\mathrm{i}=1}^{\mathrm{n}}\left(\mathrm{C}_{\mathrm{O}, \mathrm{i}}-\overline{\mathrm{C}_{\mathrm{O}}}\right)\left(\mathrm{C}_{\mathrm{E}, \mathrm{i}}-\overline{\mathrm{C}_{\mathrm{E}}}\right)}{\sqrt{\sigma_{\mathrm{O}} \sigma_{\mathrm{E}}}}\right]$ 
$211 \quad \mathrm{FV}=2 \frac{\sigma_{\mathrm{O}-\sigma_{\mathrm{E}}}}{\sigma_{\mathrm{O}}+\sigma_{\mathrm{E}}}$

212 where $n=$ the total number of observations, $C_{o, i}=$ the $i$ th observed value, $C_{E, i}=$ the $i$ th estimated value and

$213 \overline{C_{O}}$ and $\overline{C_{E}}$ are the observation and estimation averages, respectively. These indicators were calculated in 214 both development and validation steps.

\section{3. Results and discussion}

\section{3.1. Levels of the studied metals}

218 Fig. 3 shows that the $\mathrm{Pb}$ and $\mathrm{Ni}$ mean concentrations for the period of study are below the corresponding

219 legislated objective/limit values for ambient air. Moreover, the Mn average concentration is also below the 220 WHO air quality guideline in relation to manganese. Nevertheless, there are some particular cases, i.e., Ni 221 and $\mathrm{Mn}$, where the non-averaged concentrations (individual sample concentrations) of these pollutants 222 amply exceed the corresponding objective/limit values as follows: as shown, the Ni and Mn maximum 223 observed concentrations exceed by 10 and 6 times their LAT and LAT-equivalent values, respectively. 224 Special attention should be paid to model performance in this sense because exposures to high levels of 225 these metals may have detrimental effects on human health. It has been demonstrated that inhaled 226 manganese produces neurotoxic effects that vary from neuropsychological and motor functions (Mergler 227 et al. 1999), postural stability (Hernández-Bonilla et al. 2011) and increased risk of Parkinson’s disease 228 (Finkelsteinn and Jerret 2007) at lower concentration exposures (near $50 \mathrm{ng} \mathrm{m}^{-3}$ ) to a movement disorder 229 known as Manganism at concentrations above $1 \mathrm{mg} \mathrm{m}^{-3}$ (Aschner et al. 2005). Regarding vanadium, its 230 toxic effects depend on its degree of oxidation and may include irritation of the respiratory tract, 231 haematological and biochemical changes and functional lesions in certain organs (Sumanta et al. 2015).

232 The studies conducted by Hleis (2010) and Kfoury (2013) have shown that the levels of Pb, Ni, Mn, V, Cr 233 and other metals and metalloids in Dunkerque are remarkably associated with industry as they have been 234 reported to be tracers of local industrial activities. The results of pollution roses and receptor modelling for 235 source apportionment by means of non-negative matrix factorization indicate that $\mathrm{Pb}$ emissions may be 236 mainly attributed to integrated steelworks, which is an Mn emission source as well (Kfoury 2013; Hleis 237 2010). However, the ferromanganese manufacturing plant emissions also influence the levels of $\mathrm{Pb}$ and 238 certainly the levels of Mn (Hleis 2010). Ni and V are tracers of heavy oil combustion because they explain 
$23972 \%$ and $86 \%$ of the observed concentrations, respectively (Kfoury 2013). With regard to Cr, it is 240 considered to be a marker of the activity of the electric steel plant, although Cr levels may also be partly 241 due to oil combustion. The strong presence of industrial activities in Dunkerque and the firm connection 242 between ambient air metal levels and the local industry makes the city a suitable location to develop

243 objective estimation models for metals because the inputs to these models partly consist of major pollutant 244 concentrations, which are undoubtedly related to industry as well.

246 3.2. Estimation of $\mathrm{Pb}$ and $\mathrm{Ni}$

247 Table 1 presents the results obtained with the best developed models for the two considered EU-regulated 248 metals $(\mathrm{Pb}$ and $\mathrm{Ni})$ using the three different considered approaches as follows: PLSR, ANNs and PCA249 ANN. Results related to training (T) and external validation (V) subsets are presented for each model.

250 In the first place, for the evaluation of these models as an air quality assessment tool from a regulatory point 251 of view, which is the main goal of the present study, the mean value estimation and the conformity of the compliance with the uncertainty requirements are two key aspects to take into account. In this regard, while

253 complying with the uncertainty requirements (expressed in this study in terms of the RME and RDE indices)

254 because both uncertainty indices are well below $100 \%$, which is the maximum uncertainty percentage 255 allowed by the EU for objective estimation techniques to be used for air quality assessment, every model 256 is able to provide a good estimate of the mean concentration due to the lower values obtained for the FB

257 index, which is an indicative measure of the accuracy in estimating mean values. For the training stage, the 258 PLSR FB index values are lower than the FB values with ANNs and the PCA-ANN model. The reason why 259 the FB index of PLSR models is so small is that the PLSR-estimated and observed mean values are nearly 260 equal, resulting in an FB index very close to its ideal value, which, according to Eq. (11), is 0. However, if 261 attention is to be paid to the values of the rest of the FB indices, it is evident that they are not significantly 262 higher as the estimated mean values are close to the corresponding observed values in every case.

263 It is worth noting that the mean values in this work are not in fact annual mean concentrations because the 264 available data samples belonged to a period of study limited to three months, from mid-February to mid-

265 May 2008. The sampling period varied from 6 to 14 hours, and consequently, the levels of pollutants 266 presented significant variability. Therefore, there was an additional difficulty for the estimation.

267 Although a correct estimation of the mean value while fulfilling the EU uncertainty requirements is 268 sufficient for a model application in the frame of the EU Air Quality Directive, it would be greatly 
269 preferable for the model to also be able to correctly estimate the individual sample concentrations. To 270 evaluate this capacity and provide a more comprehensive response of model performances, a series of 271 additional statistical indicators have been addressed. With regard to these performance indices, the 272 correlation coefficient values of the PLSR and ANN models are within the range of 0.5-0.9, indicating a 273 good tendency of the estimated and observed values to vary together. Nevertheless, even if the $r$ values are 274 close to 1 , there is no guarantee that the estimated and observed values match each other, only that they 275 may differ by a consistent factor. For this reason, other statistics must be taken into consideration.

276 As for PCA-ANN models, they provide lower values of the correlation coefficient - within the range of 0.3-

277 0.6- than PLSR and ANNs. This fact, together with an increase in uncertainty indices, indicates that, for 278 this specific application, performing PCA prior to the development of ANNs is not an effective alternative.

279 Models have been evaluated on the basis of comparisons against observations via a set of statistical 280 indicators, which, while providing insight on general model performance, do not necessarily indicate 281 whether model results have reached a sufficient quality level for a given application, e.g., for policy support. 282 Ideally, models should have a correlation coefficient close to 1 and FB, RMSE and FV values close to 0. 283 Unfortunately, in practice, due to the uncertainty of observation and the analytical determination in the 284 laboratory, these values will rarely be achieved. In this regard, Kumar et al. (1993) propose values for some 285 of these parameters associated with a minimum quality for the models: NMSE $\leq 0.5$ and $-0.5 \leq \mathrm{FB} \leq 0.5$.

286 A supplementary manner to evaluate model performance is by means of a graphic representation. In this 287 regard, Fig. 4 depicts the estimated normalized concentrations of $\mathrm{Pb}$ and Ni obtained with the PLSR and 288 ANN models for the training and external validation subsets. As observed, both models exhibit difficulties 289 in accurately estimating the individual sample concentrations, leading to an underestimation of the highest 290 concentrations. Notwithstanding, PLSR and ANNs capture the underlying trend during training, although 291 there is a slightly better fitting when using ANNs, as reflected in the lower RMSE and FV values obtained 292 with ANNs with respect to those obtained with the PLSR model.

293 With respect to the external validation subset, as a result of a decrease in the accuracy of the estimations, 294 there is a general slight decrease in the correlation coefficient values and an increase in the values of the 295 RMSE and FV indices of every model compared with those obtained for the training subset. However, the 296 FB index values for PLSR and ANNs are below 0.5 and, therefore, within the acceptable range for FB for 297 an air quality model suggested by Kumar et al. (1993). Additionally, for the PLSR and ANN models, the 298 correlation coefficient of the external validation subset ranged from 0.5-0.8, which was similar to those 
299 obtained for the training subset. Consequently, based on the performance results obtained for external 300 validation, PLSR and ANNs may be considered proper approaches to estimate ambient $\mathrm{Pb}$ and $\mathrm{Ni}$ levels in 301 the studied site. Nevertheless, as a general remark, the best pair of training and external validation statistics

302 are found when using ANNs, which indicates that the model is able to not only fit the data correctly but 303 also provide good estimates of data not used for the development of the models, which implies that ANNs 304 present better generalization ability than the other studied techniques.

\subsection{Estimation of $\mathrm{Mn}, \mathrm{V}$ and $\mathrm{Cr}$}

307 The results of the best developed models for $\mathrm{Mn}, \mathrm{V}$ and $\mathrm{Cr}$ are presented in Table 2. Despite the fact that 308 the ambient air levels of these pollutants are not regulated by the European Directives, the model evaluation 309 analysis is performed in the same manner as in the case of $\mathrm{Pb}$ and $\mathrm{Ni}$. Nevertheless, because these pollutants 310 are lacking a policy limit/target value, an $\mathrm{RDE}$-equivalent $\left(\mathrm{RDE}_{\mathrm{eq}}\right)$ uncertainty index has been calculated 311 based on the version equivalent to LAT values for regulated pollutants, as mentioned in section 2.4. Note 312 that, with this assumption, $\mathrm{Mn}, \mathrm{V}$ and $\mathrm{Cr}$ mean values, unlike those of $\mathrm{Pb}$ and $\mathrm{Ni}$, are closer to their 313 corresponding LAT.

314 As can be observed, the EU uncertainty requirements for objective estimation techniques are fulfilled with 315 an RME and an $\mathrm{RDE}_{\text {eq }}$ lower than $100 \%$ in all cases, except for the external validation RME index of the 316 Cr PCA-ANN model. However, it could be argued that because some of these metals may present 317 considerably higher air concentrations, such as Mn, which exceeds by almost six times $150 \mathrm{ng} \mathrm{m}^{-3}$ (which 318 is the WHO air quality guideline used as LAT-equivalent) with a maximum observed value of $872.8 \mathrm{ng} \mathrm{m}^{-}$

$319{ }^{3}$ for the period of study (Fig. 3), more restrictive uncertainty requirements should be addressed, considering 320 that an allowed $100 \%$ uncertainty in the estimation may lead to erroneously regarding as acceptable an 321 underestimation of a potentially dangerous pollutant level.

322 With respect to the mean concentration, lower values of the FB index for the Mn, V and Cr PSLR and ANN 323 models indicate acceptable training and external validation estimations. These values are within the same 324 order of magnitude as those obtained for $\mathrm{Pb}$ and $\mathrm{Ni}$ and below 0.5 , complying with the minimum quality 325 requirements proposed by Kumar et al. (1993). Again, that is not applicable to Cr PCA-ANN models with 326 an external validation FB index of -1.46 .

327 As for the models' performance in relation to the estimation of individual sample concentrations, 328 correlation coefficient values lower than 0.66 for external validation indicate an unsatisfactory fitting. 
329 However, this measure can be dominated by a small proportion of extreme values that may not reflect the 330 behaviour of the bulk of the data. At any rate, the ANN model correlation coefficients are greater than those 331 obtained for the PLSR and PCA-ANN models. This, together with the fact that ANNs provide the lowest 332 FB index and adequate RME and $\mathrm{RDE}_{\mathrm{eq}}$, points to ANNs as the most suitable approach of the three studied.

\section{4. Conclusions}

335 In this work, statistical models based on PLSR and ANNs to estimate the levels of the considered EU336 regulated metals, i.e., $\mathrm{Pb}$ and $\mathrm{Ni}$, have been developed and externally validated. Based on the results 337 obtained and according to the European Air Quality Framework Directive, these models can be taken into 338 consideration as valid approaches to be used as objective estimation techniques for air quality assessment 339 in relation to metals in the area of study because they are able to correctly estimate mean values within an 340 uncertainty range up to $100 \%$. Both linear (PLSR) and non-linear (ANNs) statistical models show a 341 comparable performance, although the latter exhibit an enhanced generalization ability. However, ANN 342 performance experienced no improvements by the application of factor analysis techniques, such as PCA, 343 before model development.

344 Additionally, in this study, some metals that lack a limit/target value in European legislation, namely Mn,

$345 \mathrm{~V}$ and $\mathrm{Cr}$, have also been considered due to the strong relationship that exists between their levels and the 346 local industry of the study area and due to the scientific evidence that suggests that some of these non347 regulated metals can also cause damage to human health. As with $\mathrm{Pb}$ and $\mathrm{Ni}$, the PLSR and ANN models 348 for Mn and V work relatively well in terms of mean estimation within the EU Directive uncertainty limits.

349 Nevertheless, they are not able to properly describe variations of $\mathrm{Cr}$.

350 Finally, the statistical models developed for every metal struggle with the estimation of the individual 351 sample concentrations and, as with many deterministic models, tend towards a slight underestimation.

352 Therefore, further work will focus on deepening knowledge regarding the interactions between the different 353 inputs and their relationship with the outputs to improve this specific matter.

\section{Acknowledgements}

356 This work was supported by the Spanish Ministry of Economy and Competitiveness (MINECO) through the Projects CTM2010-16068/CTM2013-43904R and the FPI short stay EEBB-I-13-07691. Germán Santos

358 would also like to thank the Unité de Chimie Environnementale et Interactions sur le Vivant (UCEIV) at 
La Maison de la Recherche en Environnement Industriel for welcoming him as a guest PhD student in their 360 facilities.

References

363 Abdi H (2010) Partial Least Squares regression and Projection on Latent Structure regression (PLS regression). Wiley Interdiscip Rev Comput Stat 2:97-106

Aschner M, Erikson KM, Dorman DC (2005) Manganese dosimetry: species differences and implications 
388 Gardner MW, Dorling SR (1999) Neural network modelling and prediction of hourly $\mathrm{NO}_{\mathrm{x}}$ and $\mathrm{NO}_{2}$ 389 concentrations in urban air in London. Atmos Environ 33:709-719

390 Hleis D (2010) Evaluation de la contribution d'émissions sidérurgiques à la teneur en particules en 391 suspension dans l'atmosphère à une échelle locale. Dissertation, Université du Littoral Côte d'Opale

392 Karar K, Gupta AK (2006) Seasonal variations and chemical characterization of ambient $\mathrm{PM}_{10}$ at residential 393 and industrial sites of an urban region of Kolkata. Atmos Res 81:36-53

394 Kfoury A (2013) Origin and physicochemical behaviour of atmospheric $\mathrm{PM}_{2.5}$ in cities located in the littoral 395 area of the Nord-Pas-de-Calais region, France. Dissertation, Université du Littoral Côte d'Opale

396 Kukkonen J, Partanen L, Karppinen A, Ruuskanen J, Junninen H, Kolehmainen M, Niska H, Dorling S, 397 Chatterton T, Foxall R, Cawley G (2003) Extensive evaluation of neural network models for the 398 prediction of $\mathrm{NO}_{2}$ and $\mathrm{PM}_{10}$ concentrations, compared with a deterministic modelling system and

400 Kumar A, Luo J, Bennett G (1993) Statistical evaluation of lower flammability distance (LFD) using four 401 hazardous release models. Process Saf Prog 12:1-11

402 Lu WZ, Wang WJ, Wang XK, Xu ZB, Leung AYT (2003) Using improved neural network model to analyse 403 RSP, $\mathrm{NO}_{\mathrm{X}}$ and $\mathrm{NO}_{2}$ levels in urban air in Mong Kok, Hong Kong. Environ Monit Assess 87:235-254 404 Mergler D, Baldwin M, Belanger S, Larribe F, Beuter A, Bowler R et al. (1999) Manganese neurotoxicity, 405

406 Perez P, Reyes J (2002) Prediction of maximum of 24-h average of $\mathrm{PM}_{10}$ concentrations $30 \mathrm{~h}$ in advance in 407

408 Pires JCM, Martins FG, Sousa SIV, Alvim-Ferraz MCM, Pereira MC (2008) Prediction of the daily mean 409

410 Polat K, Durduran SS (2012) Usage of output-dependent data scaling in modelling and prediction of air 411 pollution daily concentration values $\left(\mathrm{PM}_{10}\right)$ in the city of Konya. Neural Comput Appl 21:2153-2162 412 Singh KP, Gupta S, Kumar A, Shukla SP (2012) Linear and nonlinear modelling approaches for urban air 413 quality prediction. Sci Total Environ 426:244-255

414 Sousa SIV, Martins FG, Alvim-Ferraz MCM, Pereira MC (2007) Multiple linear regression and artificial 415 neural networks based on principal components to predict ozone concentrations. Environ Modell Softw 416 22:97-103 
417 Sumanta KG, Rumpa S, Bidyut S (2015). Toxicity of inorganic vanadium compounds. Res Chem Intermed $418 \quad 41: 4873-4897$

419 Thorpe A, Harrison RM (2008) Sources and properties of non-exhaust particulate matter from road traffic: 420 a review. Sci Total Environ 400:270-282

421 Ul-Saufie AZ, Yahaya AS, Ramli NA, Rosaida N, Hamid HA (2013) Future daily PM 10 concentrations 422 prediction by combining regression models and feedforward backpropagation models with principle 423 component analysis (PCA). Atmos Environ 77:621-630

424 WHO (2000) Air quality guidelines for Europe $2^{\text {nd }}$ edition. World Health Organisation, Geneva 425 Wold S, Sjöström M, Eriksson L (2001) PLS-regression: A basic tool of chemometrics. Chemom Intell Lab 426 Syst 58:109-130 


\section{$427 \quad$ Figure captions}

428

429 Figure 1: Structure of the artificial neural network

430

431 Figure 2: Sampling site and monitoring and meteorological stations in Dunkerque

432

433 Figure 3: Box-plot of the levels of $\mathrm{Pb}, \mathrm{Ni}, \mathrm{Mn}, \mathrm{V}$ and $\mathrm{Cr}$ for the period of study. The box extends between

434 the upper and lower quartiles with the inner line representing the median value. The whiskers indicate the 435 minimum and maximum values.

436

437 Figure 4: Comparison between the observed and modelled normalized $\mathrm{Pb}$ and $\mathrm{Ni}$ concentrations 
Table 1. Uncertainty, mean concentration and performance statistics for the best models developed for $\mathrm{Pb}$ and $\mathrm{Ni}$

\begin{tabular}{|c|c|c|c|c|c|c|c|c|c|c|}
\hline \multirow{2}{*}{ Metal } & \multirow{2}{*}{ Model } & \multirow{2}{*}{ Subset $^{\mathrm{a}}$} & \multicolumn{2}{|c|}{ EU Uncertainty } & \multicolumn{3}{|c|}{ Mean Concentration $^{b}$} & \multicolumn{3}{|c|}{ Performance } \\
\hline & & & RME (\%) & RDE (\%) & Co $10^{2}$ & $\mathrm{C}_{\mathrm{E}} 10^{2}$ & FB $10^{2}$ & $\mathbf{r}$ & RMSE $10^{2}$ & FV 10 \\
\hline \multirow{7}{*}{$\mathrm{Pb}$} & \multirow{2}{*}{ PLSR } & $\mathrm{T}$ & 28.1 & 1.44 & 6.52 & 6.52 & $3.710^{-08}$ & 0.823 & 3.94 & 1.95 \\
\hline & & $\mathrm{V}$ & 31.9 & 0.31 & 7.46 & 8.88 & -17.4 & 0.837 & 4.48 & -2.78 \\
\hline & \multirow{2}{*}{ ANN } & $\mathrm{T}$ & 18.3 & 2.10 & 6.38 & 6.84 & -7.0 & 0.932 & 2.72 & -0.85 \\
\hline & & $\mathrm{V}$ & 54.0 & 0.54 & 7.46 & 8.31 & -10.8 & 0.861 & 4.90 & -4.12 \\
\hline & \multirow{3}{*}{ PCA-ANN } & $\mathrm{T}$ & 40.1 & 2.22 & 6.57 & 6.78 & -3.2 & 0.663 & 3.90 & 5.82 \\
\hline & & & & & & & & & & \\
\hline & & $\mathrm{V}$ & 90.4 & 1.38 & 3.69 & 7.95 & -73.1 & 0.266 & 5.64 & -2.56 \\
\hline \multirow{7}{*}{$\mathrm{Ni}$} & \multirow[b]{2}{*}{ PLSR } & $\mathrm{T}$ & 65.9 & 12.87 & 68.5 & 68.5 & $-7.510^{-11}$ & 0.560 & 80.5 & 5.64 \\
\hline & & $\mathrm{V}$ & 83.6 & 11.70 & 156.6 & 98.2 & 45.8 & 0.556 & 241.3 & 14.62 \\
\hline & \multirow[b]{2}{*}{ ANN } & $\mathrm{T}$ & 29.2 & 18.55 & 73.4 & 73.8 & -0.5 & 0.873 & 54.2 & 1.44 \\
\hline & & $\mathrm{V}$ & 50.0 & 17.60 & 156.6 & 115.8 & 30.0 & 0.702 & 186.6 & 4.77 \\
\hline & \multirow{3}{*}{ PCA-ANN } & $\mathrm{T}$ & 64.9 & 24.86 & 95.9 & 95.8 & 0.1 & 0.470 & 161.1 & 7.20 \\
\hline & & & & & & & & & & \\
\hline & & V & 42.6 & 2.50 & 68.6 & 94.9 & -32.2 & 0.443 & 63.2 & -3.04 \\
\hline
\end{tabular}

a T: Training; V: Validation

${ }^{\mathrm{b}}$ O: Observed; E: Estimated 
Table 2. Uncertainty, mean value and performance statistics for the best models developed for $\mathrm{Mn}, \mathrm{V}$ and $\mathrm{Cr}$

\begin{tabular}{|c|c|c|c|c|c|c|c|c|c|c|}
\hline \multirow{2}{*}{ Metal } & \multirow{2}{*}{ Model } & \multirow{2}{*}{ Subset $^{\mathrm{a}}$} & \multicolumn{2}{|c|}{ EU Uncertainty } & \multicolumn{3}{|c|}{ Mean Concentration $^{b}$} & \multicolumn{3}{|c|}{ Performance } \\
\hline & & & RME (\%) & $\operatorname{RDE}_{\text {eq }}(\%)$ & Co $10^{2}$ & $C_{E} 1^{2}$ & FB $10^{2}$ & $\mathbf{r}$ & RMSE $10^{2}$ & FV 10 \\
\hline \multirow{6}{*}{$\mathrm{Mn}$} & \multirow{2}{*}{ PLSR } & $\mathrm{T}$ & 53.9 & 4.71 & 32.55 & 33.43 & -2.7 & 0.580 & 41.49 & 3.57 \\
\hline & & $\mathrm{V}$ & 53.1 & 50.23 & 64.95 & 58.24 & 10.9 & 0.184 & 92.80 & 6.99 \\
\hline & \multirow{2}{*}{ ANN } & $\mathrm{T}$ & 52.6 & 60.51 & 33.61 & 21.38 & 44.5 & 0.704 & 39.20 & 3.42 \\
\hline & & $\mathrm{V}$ & 48.2 & 68.86 & 64.95 & 63.77 & 1.8 & 0.457 & 81.56 & 4.64 \\
\hline & \multirow{2}{*}{ PCA-ANN } & $\mathrm{T}$ & 66.4 & 78.51 & 46.05 & 46.05 & 0.0 & 0.463 & 64.77 & 7.34 \\
\hline & & V & 35.3 & 11.62 & 29.49 & 30.33 & -2.8 & 0.431 & 42.72 & 1.74 \\
\hline \multirow{6}{*}{ V } & \multirow{2}{*}{ PLSR } & $\mathrm{T}$ & 42.7 & 1.20 & 13.12 & 13.42 & -2.3 & 0.694 & 8.17 & 2.33 \\
\hline & & $\mathrm{V}$ & 31.5 & 4.74 & 18.07 & 18.48 & -2.2 & 0.590 & 11.21 & 3.16 \\
\hline & \multirow{2}{*}{ ANN } & $\mathrm{T}$ & 41.6 & 4.28 & 14.27 & 14.00 & 1.9 & 0.806 & 7.19 & 1.78 \\
\hline & & V & 30.7 & 5.45 & 18.07 & 18.45 & -2.1 & 0.663 & 10.43 & 2.11 \\
\hline & \multirow{2}{*}{ PCA-ANN } & $\mathrm{T}$ & 42.9 & 1.60 & 13.14 & 13.20 & -0.5 & 0.747 & 5.91 & 2.93 \\
\hline & & V & 12.5 & 15.60 & 12.79 & 16.50 & -25.4 & 0.366 & 13.97 & 0.56 \\
\hline \multirow{6}{*}{$\mathrm{Cr}$} & \multirow{2}{*}{ PLSR } & $\mathrm{T}$ & 88.8 & 1.01 & 4.58 & 3.96 & 14.6 & -0.031 & 15.28 & 11.34 \\
\hline & & V & 78.5 & 25.74 & 6.97 & 5.78 & 18.7 & 0.077 & 18.09 & 12.78 \\
\hline & \multirow{2}{*}{ ANN } & $\mathrm{T}$ & 50.0 & 39.17 & 5.59 & 3.25 & 53.1 & -0.040 & 19.24 & 6.53 \\
\hline & & V & 83.6 & 27.89 & 6.97 & 3.55 & 65.0 & -0.240 & 19.06 & 14.76 \\
\hline & \multirow{2}{*}{ PCA-ANN } & $\mathrm{T}$ & 79.2 & 0.43 & 7.15 & 7.90 & -10.0 & 0.331 & 18.33 & 9.70 \\
\hline & & V & 489.9 & 0.43 & 1.45 & 9.31 & -146.3 & 0.275 & 10.04 & -13.57 \\
\hline
\end{tabular}

a T: Training; V: Validation

${ }^{\text {b }}$ O: Observed; E: Estimated 

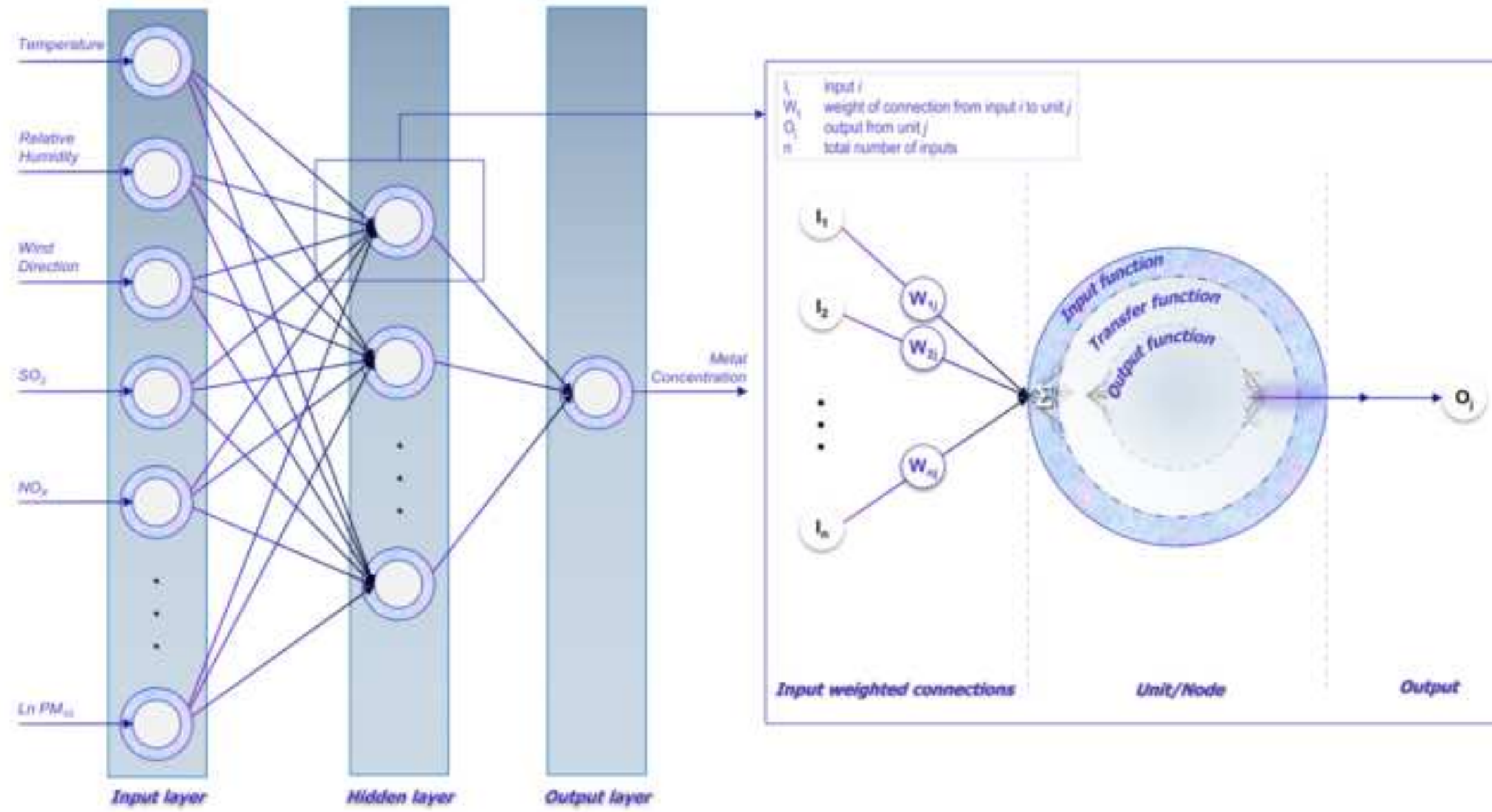

Wi meight of connection fom nest i a vit;

Q. outcuthom uni Hodos kayer

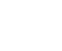




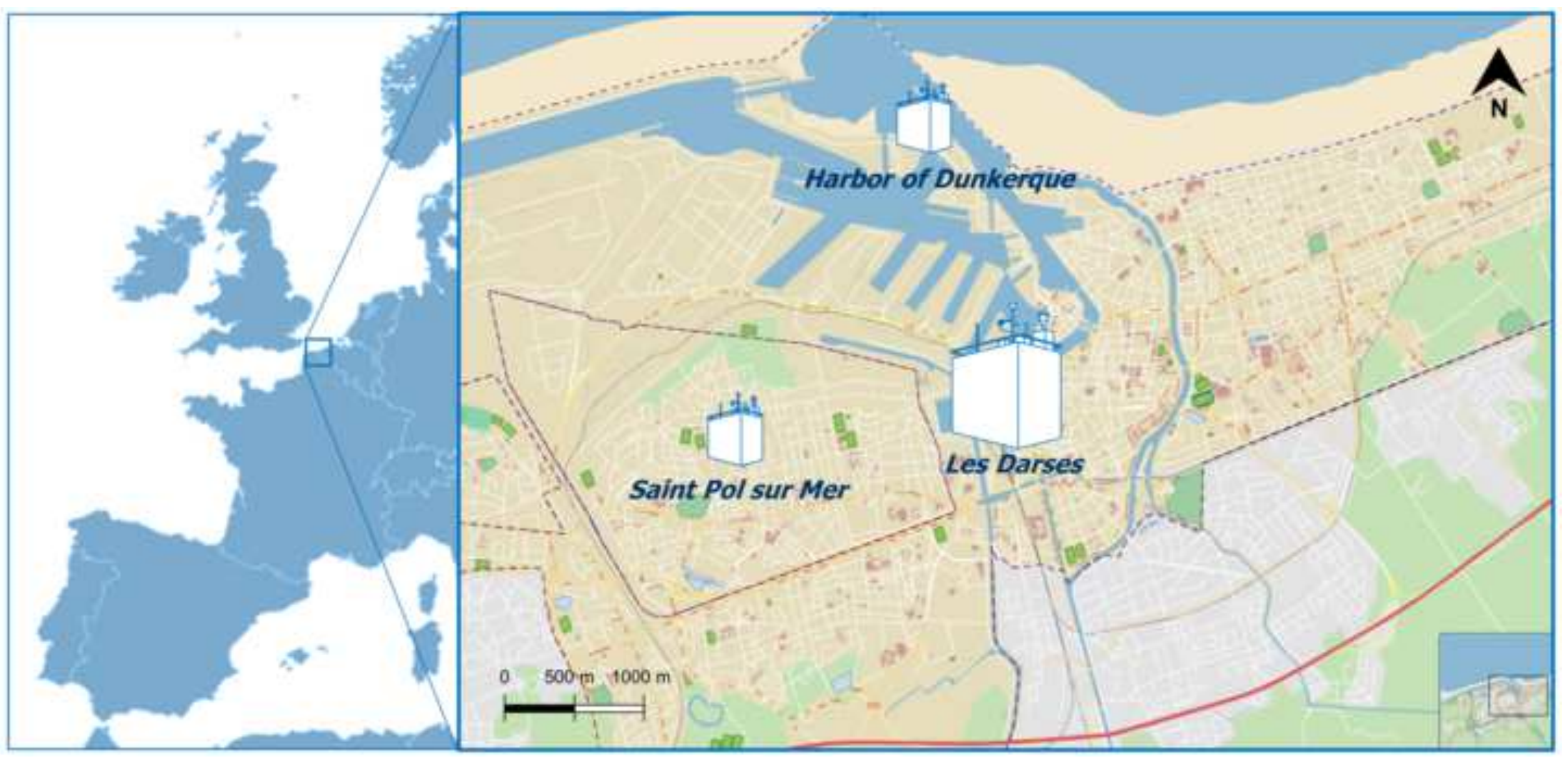




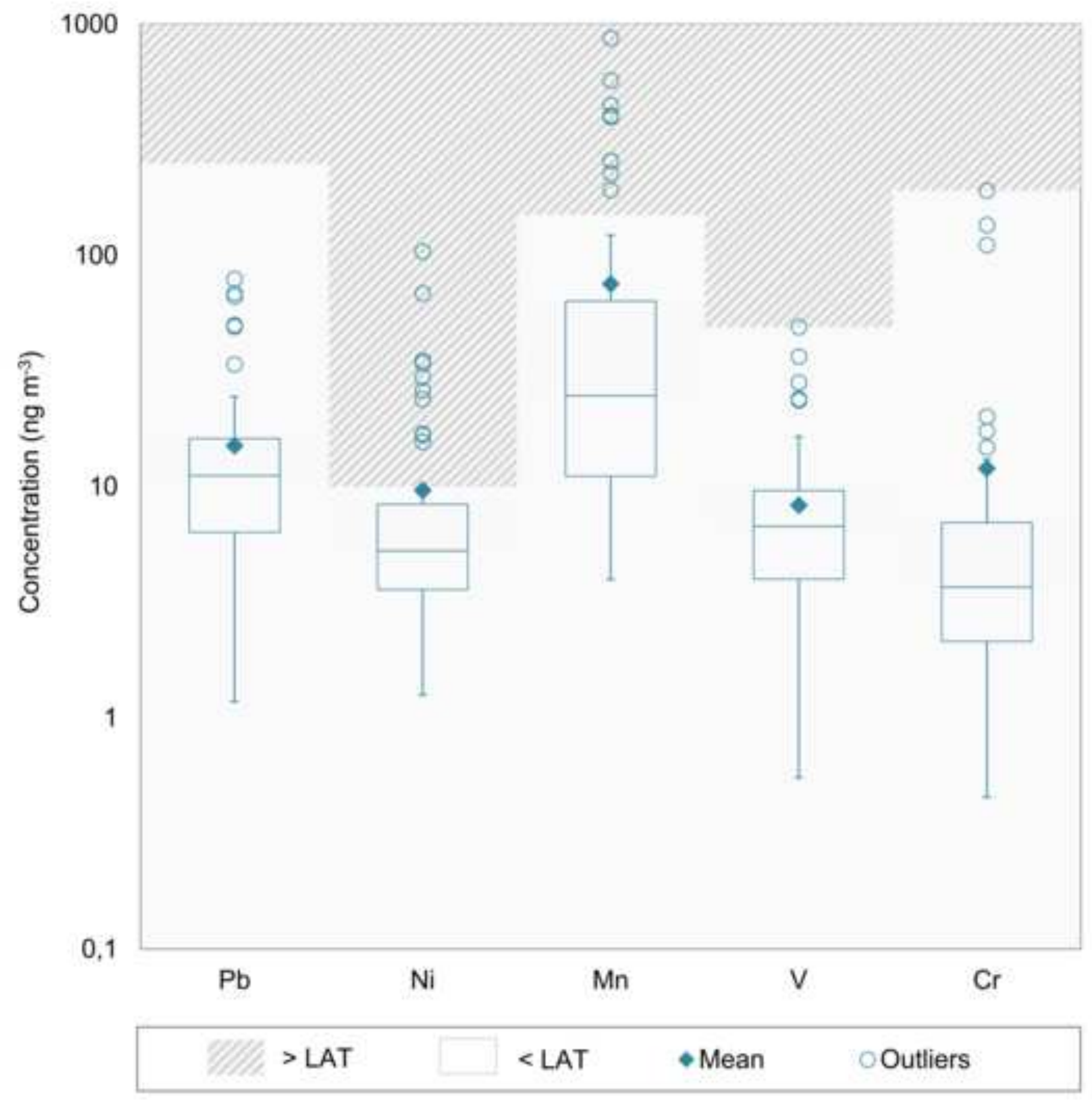



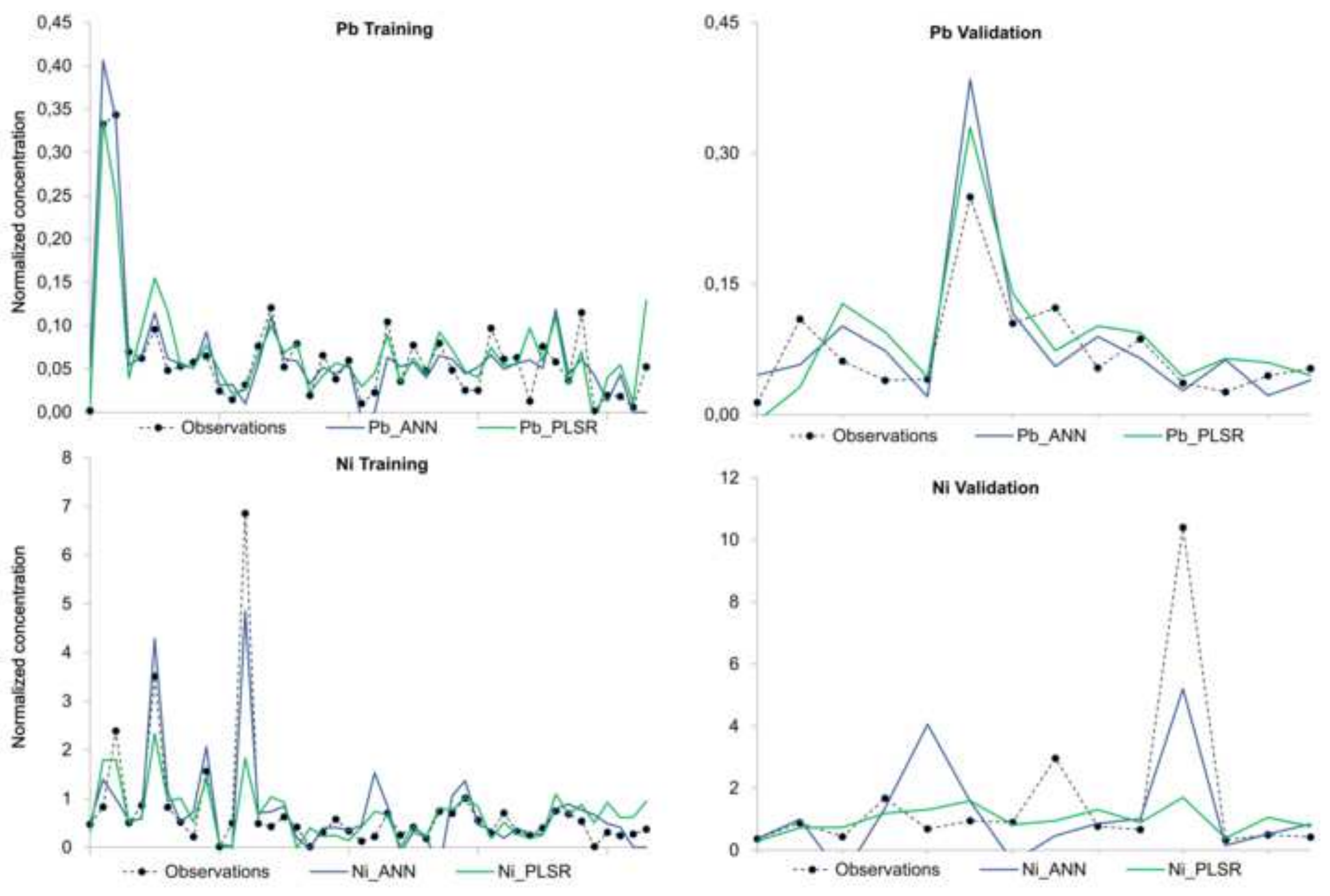

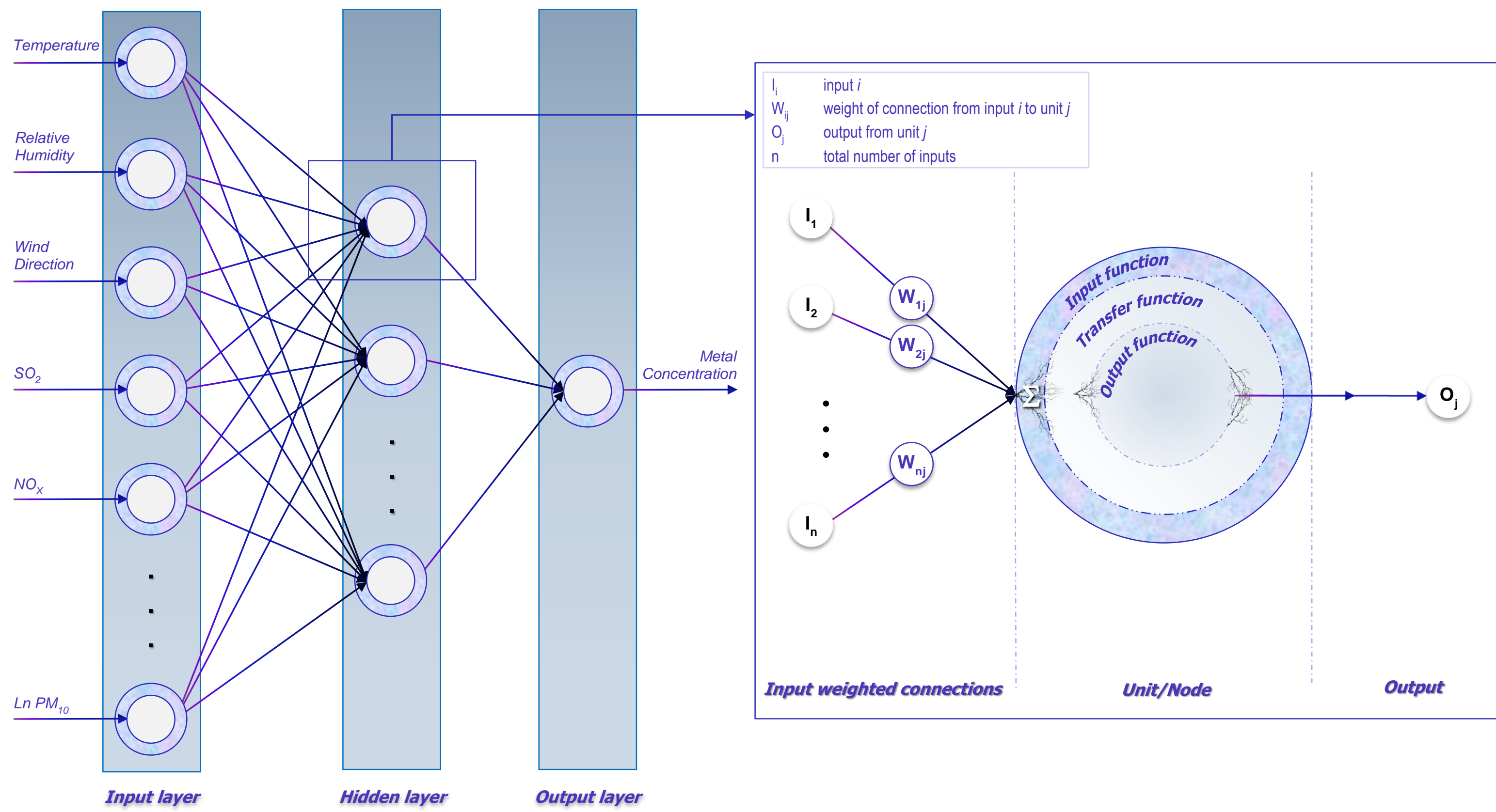

Input weighted connections

Unit/Node

utput

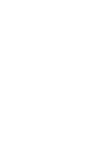

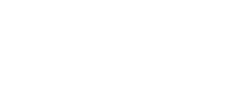




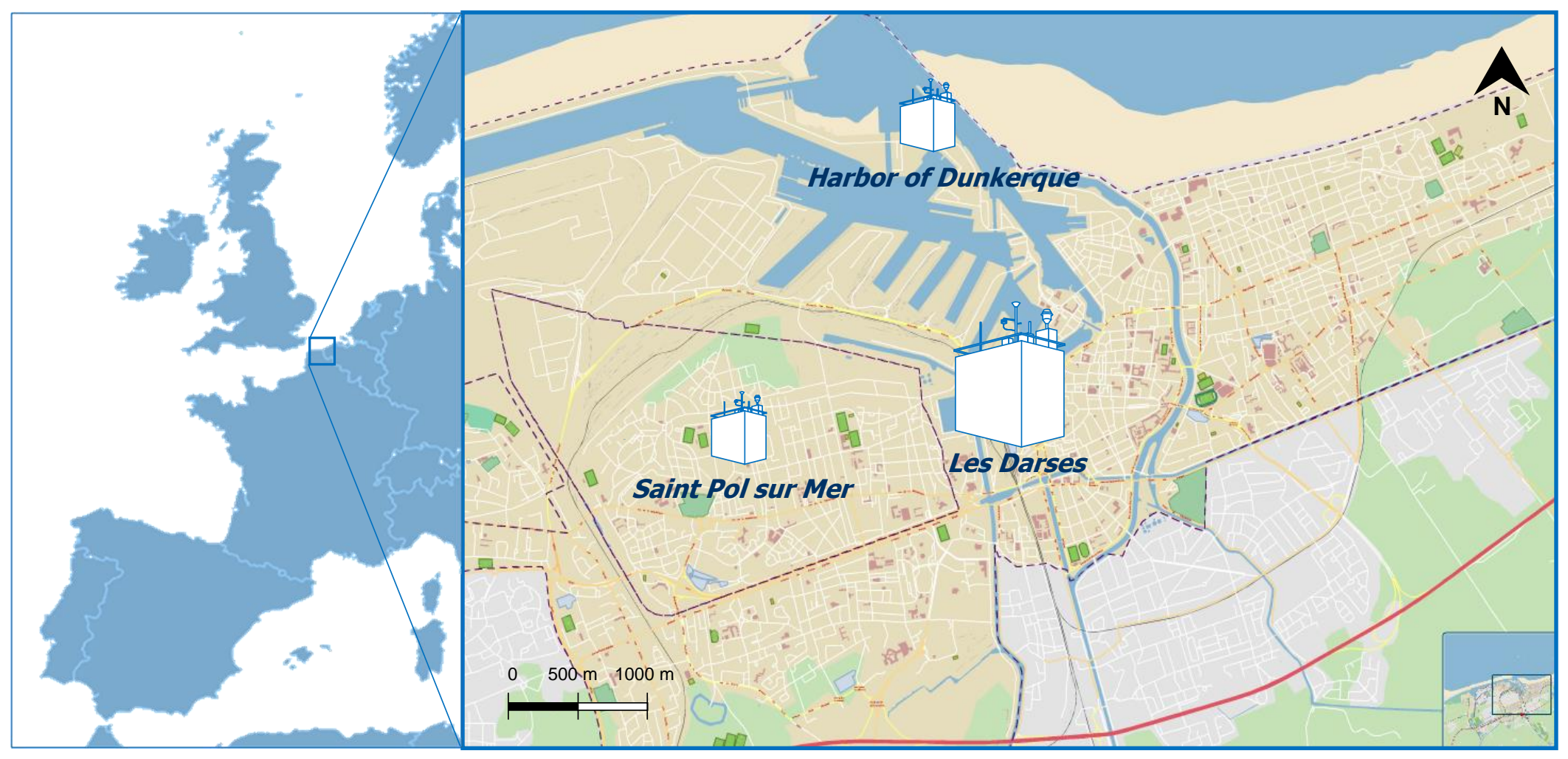




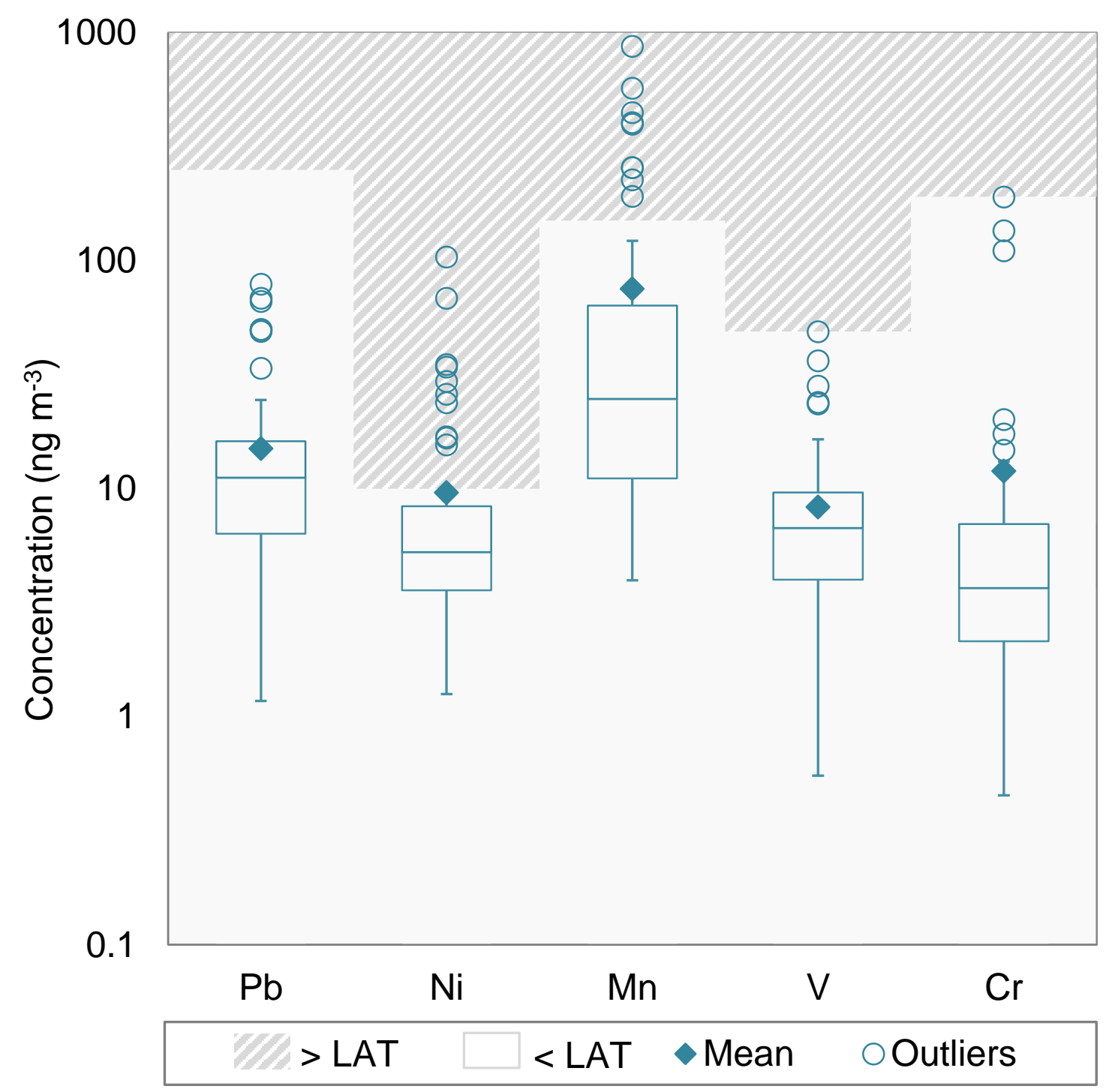

